\title{
Why women prefer epidural analgesia during childbirth: The role of beliefs about epidural analgesia and pain catastrophizing
}

\author{
Eva Van den Bussche ${ }^{a}$, Geert Crombez ${ }^{\text {b,c,* }}$, Christopher Eccleston ${ }^{\mathrm{d}}$, \\ Michael J.L. Sullivan ${ }^{\mathrm{e}}$ \\ ${ }^{a}$ Faculty of Psychology and Educational Sciences, University of Leuven - Campus Kortrijk, Belgium \\ ${ }^{\mathrm{b}}$ Department of Experimental-Clinical and Health Psychology, Ghent University, Henri Dunantlaan 2, B-9000 Ghent, Belgium \\ ${ }^{\mathrm{c}}$ Research Institute for Psychology and Health, The Netherlands \\ d Pain Management Unit, Royal National Hospital for Rheumatic Disease and The University of Bath, Bath, UK

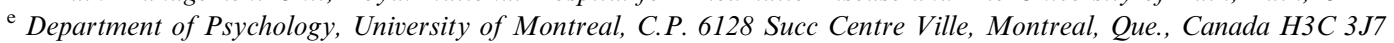

Received 21 September 2005; received in revised form 3 February 2006; accepted 2 March 2006

Available online 18 April 2006

\begin{abstract}
This study investigated the reasons that might lead women to choose or not choose epidural analgesia as a strategy for the management of pain in childbirth. In our sample $55 \%$ of 114 women chose EA. Logistic regression resulted in a statistical model with four unique and independent predictors: Parity status and the fear of the side effects of EA each reduced the odds of choosing EA by half, whereas the desire to have a pain-free childbirth and positive experiences with EA of family and friends each doubled the odds of choosing EA. Pain catastrophizing was not related to EA use. The lack of an interrelationship between pain catastrophizing and EA use is probably due to an ambivalent attitude towards EA in pain catastrophizers. Pain catastrophizing was positively associated with the fear of being overwhelmed by labour pain and tendencies to avoid the pain, but also positively with the fear of pain during the insertion of the EA needle. Pain catastrophizing was also strongly related to recommendations to use EA from others, in particular from the midwife and from the gynecologist. Results are discussed in terms of the social impact of pain catastrophizing. (C) 2006 European Federation of Chapters of the International Association for the Study of Pain. Published by Elsevier Ltd. All rights reserved.
\end{abstract}

Keywords: Childbirth; Labour pain; Epidural analgesia; Pain catastrophizing; Beliefs

\section{Introduction}

For many women childbirth is the most painful experience encountered. When compared to other forms of acute pain, childbirth pain ranks among the most intense (Melzack, 1984). Across the ages, women have searched for pharmacological and non-pharmacological ways to manage childbirth pain (Caton, 2004), and var-

\footnotetext{
* Corresponding author. Address: Department of ExperimentalClinical and Health Psychology, Ghent University, Henri Dunantlaan 2, B-9000 Ghent, Belgium. Tel.: +329 26464 61; fax: +32 92646489. E-mail address: Geert.Crombez@UGent.be (G. Crombez).
}

ious methods for relief of childbirth pain have become established. In Western cultures the use of epidural analgesia (EA) is widespread, and its benefits are well recognized although it is not without risks (Caton et al., 2002; Leighton and Halpern, 2002; Reynolds et al., 2002). Despite the relatively positive evidence base, many women do not choose EA (Horowitz et al., 2004). Why some women favor EA is not known. This study was designed to explore the reasons that inform the choice of EA. Two theoretical models have influenced the approach to this question. First, the generic model of health related choice outlined in the Theory of Planned Behaviour (Ajzen, 1991), and second, the 
appraisal model of catastrophic thinking about pain (Sullivan et al., 2001). Although both models stem from a different background, they are not mutually exclusive.

According to the TPB, future behaviour that is under the deliberate control of the individual is best explained by the intention to perform that behaviour (intention), the specific attitudes towards the behaviour (attitudes), the influence of others about the behaviour (subjective norms), and the perceived control/ability to perform the behaviour (perceived control). The TPB has been successfully applied to a variety of health behaviours (Godin and Kok, 1996; Armitage and Conner, 2001). In line with this research, the TPB was used as a framework to measure specific opinions related to childbirth and EA, and to formulate hypotheses. More specifically, we predict that women would choose EA because of positive attitudes about having a relaxed, enjoyable childbirth, because of positive stories and advice about EA from others, and because of the fear of not being able to cope with pain. Catastrophic thinking about pain (an exaggerated negative orientation towards actual or anticipated pain experiences) is a key psychosocial variable in explanations of how adults and children experience pain, distress and disability (Keefe et al., 2004). Most often catastrophizing about pain has been considered within an appraisal model (Aldrich et al., 2000; Sullivan et al, 2000). In line with this view, pain catastrophizing has been found to be strongly related to pain-related fear, hypervigilance and avoidance behaviour in chronic pain (Vlaeyen and Linton, 2000; Goubert et al., 2004; Crombez et al., 2005). Despite the growing amount of research on pain catastrophizing, little is known about the influence of catastrophizing on decision-making. Childbirth may provide a unique and natural setting in which to investigate the various effects of pain catastrophizing. We hypothesize that those who catastrophize about pain will be more afraid of childbirth pain, and will be more inclined to avoid childbirth pain and, therefore, choose EA. We also expect that those who catastrophize about pain, will experience childbirth as more painful and distressing.

\section{Methods}

\subsection{Participants}

Three hundred pregnant women from two maternity clinics were invited to participate in a study about the attitudes, beliefs and use of epidural analgesia (EA). One hundred and fifty-four participants gave their informed consent and returned the questionnaires (response rate $=51 \%$ ). Women who were considered to have had no influence upon the choice for EA, were excluded. This was the case for 11 women who gave birth by caesarean section, and for 18 women who gave birth using a birthing pool where EA is not possible. Eleven women were also excluded because information regarding childbirth and EA $(n=7)$ was missing, or because essential demographic information was missing $(n=4)$. The final sample consisted of 114 women.

The mean age of the final sample $(n=114)$ was 28.74 (range 18-41). Most women (98.3\%) were married or cohabiting. More than half of the women $(55.3 \%)$ had undertaken post 18 years 'higher' education. For the majority of the women $(57 \%)$, it was their first pregnancy, whereas $29.8 \%$ of the women was pregnant of their second child. The total number of pregnancies ranged from 1 to 4 .

\subsection{Questionnaires}

Catastrophic thinking about pain was assessed by the Dutch version of the Pain Catastrophizing Scale (PCS; Sullivan et al., 1995; Crombez et al., 1998). This is a 13-item scale developed for both clinical and non-clinical populations. Participants are asked to reflect on past painful experiences and indicate the degree to which they experienced thoughts or feelings during pain on a 5-point Likert scale ranging from 0 ("not at all") to 4 ("always"). Examples of PCS items are "I can't seem to keep it out of my mind" or "I feel I can't stand it anymore". The Dutch version has been shown to have good reliability and validity in a student population and in a clinical population (Van Damme et al., 2002). Cronbach's alpha of the PCS in this study was 0.88 . The mean total score on the PCS for the women in this study was $16.93(\mathrm{SD}=8.53$, range $=1-33)$.

Two questionnaires were specifically designed for this study: the Beliefs about Epidural Analgesia Questionnaire and the Childbirth Experience Questionnaire. The Beliefs about Epidural Analgesia Questionnaire (BEAQ) assesses specific beliefs about epidural analgesia that might influence the decision to choose EA. It was developed following the theory of planned behaviour (Ajzen, 1991). An initial pool of 60 items was developed by two researchers. After feedback from midwifes, gynecologists and pilot tests with pregnant women, items were deleted or reworded. This resulted in a final questionnaire of 20 items. The intention to choose EA during childbirth was measured by indicating one of the three alternatives: "I will choose EA", "I will not choose EA" or "I'm not sure yet". All other items from the BEAQ used 5-point Likert scales ranging from 1 ("completely disagree") to 5 ("completely agree"). There were 11 attitude items, which measured the perceived advantages and disadvantages of (not) using epidural analgesia (e.g. "I'm afraid of the potential side effects of epidural analgesia"). Most items assess the beliefs component of the attitude, which are the expected outcomes of choosing EA. 
There were 5 subjective norm items, which assessed the influence of experts (e.g. midwife and gynecologist), and of the immediate social environment (e.g. family and friends) on the choice of EA. The items assessed both the direct influence of others (e.g. "I'm inclined to choose epidural analgesia because my gynecologist recommended it") and the indirect influence of others (e.g., "Because of the stories about contractions of other women, I'm thinking about choosing EA") (Devries et al., 1995). There were 3 perceived control items, which measured the perceived ability to cope with the pain during childbirth (e.g. "I think I am more able to tolerate pain from childbirth than other pains"). A description of all items can be found in Table 1 . Because our research focus was upon the most discriminative beliefs related to EA, statistical analyses are performed upon the individual item scores and not upon the general constructs of attitudes, subjective norms and perceived control.

The Childbirth Experience Questionnaire (CEQ) was developed in a similar way as the BEAQ. It consists of 10 items with a 5-point Likert scale ranging from 1 ("completely disagree") to 5 ("completely agree"), and assesses the experience of childbirth, more specifically the distress and pain experienced (e.g. "I expected the pain to be less frustrating"). Table 3 provides a short description of these items.

\subsection{Procedure and statistical analyses}

Women completed the BEAQ and the PCS between the 32nd and 40th week of their pregnancy and returned the questionnaire to their gynecologist or midwife before labor started. After childbirth, the women completed the CEQ.

Statistical analyses were performed with $\mathrm{R}$ (version 2.0.1) and SPSS 12.0. Inspection of the data revealed that 34 women had at least one missing value on items of the BEAQ and the CEQ. In order to avoid the loss of these cases, missing data were estimated. First, it was tested whether the missing data were missing completely at random (MCAR). The Little's MCAR test (Little and Rubin, 1989) revealed that the missing values on both questionnaires were missing completely at random (BEAQ: $\chi^{2}(223)=235.86, \quad p=.26 ; \quad$ CEQ: $\left.\chi^{2}(54)=60.66, p=.25\right)$. Second, missing values were imputed using the expectation maximization (EM) algorithm in SPSS 12.0. The EM method estimates missing values by an iterative process. Each iteration consists of a step to calculate expected values of parameters, and a step to calculate maximum likelihood estimates (Dempster et al., 1977). Third, results from statistical analyses using the imputed data were compared with the results from statistical analyses using listwise deletion. The pattern of results was similar.

Table 1

Means $(M)$ and standard deviations (SD) for differences in beliefs between women who went through childbirth with or without epidural analgesia (EA) and Pearson correlations between the Beliefs about Epidural Analgesia Questionnaire items and pain catastrophizing

\begin{tabular}{|c|c|c|c|}
\hline Items of the Beliefs about Epidural Analgesia Questionnaire & $M(\mathrm{SD})$ with EA & $M(\mathrm{SD})$ without EA & PCS \\
\hline \multicolumn{4}{|l|}{ Attitudes } \\
\hline EA is comfortable ${ }^{a}$ & $4.12(0.85)$ & $3.61(1.02)^{* *}$ & .16 \\
\hline I want a pain-free childbirth ${ }^{\mathrm{a}}$ & $3.54(1.20)$ & $2.55(1.46)^{* * *}$ & .18 \\
\hline I wish to fully enjoy the childbirth & $3.39(1.44)$ & $2.25(1.32)^{* * *}$ & $.22^{*}$ \\
\hline I fear the pain during the insertion of the EA needle & $2.91(1.47)$ & $2.66(1.46)$ & $.19^{*}$ \\
\hline I think I won't be able to press during the delivery with EA & $1.86(1.16)$ & $1.86(1.20)$ & .03 \\
\hline I'm willing to tolerate more pain for a child & $3.51(1.14)$ & $3.80(1.15)$ & -.15 \\
\hline I choose EA to have a more relaxed childbirth & $3.12(1.21)$ & $2.43(1.42)^{* *}$ & .15 \\
\hline I'm afraid of the potential side effects of EA & $2.70(1.45)$ & $3.41(1.30)^{* *}$ & .17 \\
\hline Suffering pain during childbirth is needless & $3.35(1.11)$ & $2.65(1.21)^{* *}$ & $.26^{* *}$ \\
\hline EA is often applied, so it probably has no disadvantages & $3.40(1.11)$ & $2.89(1.10)^{*}$ & .01 \\
\hline If I don't choose EA, I fear I'll be overwhelmed by the pain & $3.21(1.17)$ & $2.55(1.15)^{* *}$ & $.33^{* * *}$ \\
\hline \multicolumn{4}{|l|}{ Subjective norms } \\
\hline Because of the stories about contractions of other women, I'm thinking about choosing EA ${ }^{\mathrm{a}}$ & $3.16(1.18)$ & $2.23(1.44)^{* * *}$ & .01 \\
\hline Information about EA from the hospital has convinced me to choose EA & $2.36(1.11)$ & $1.71(1.07)^{* *}$ & .13 \\
\hline I'm inclined to choose EA because my midwife recommended it & $2.78(1.38)$ & $2.74(1.51)$ & $.27^{* *}$ \\
\hline Because of positive experiences with EA of family and friends, I'm thinking about choosing EA ${ }^{a}$ & $2.78(1.30)$ & $1.90(1.00)^{* * *}$ & .13 \\
\hline I'm inclined to choose EA because my gynecologist recommended it ${ }^{\mathrm{a}}$ & $3.24(1.23)$ & $3.04(1.54)$ & $.22^{*}$ \\
\hline \multicolumn{4}{|l|}{ Perceived control } \\
\hline I have confidence in my ability to tolerate labour pain & $2.92(1.25)$ & $3.51(1.14)^{*}$ & -.14 \\
\hline I think I'll be able to give birth without EA & $3.14(1.12)$ & $3.74(1.09)^{* *}$ & -.14 \\
\hline I think I am more able to tolerate pain from childbirth than other pains & $3.67(0.92)$ & $3.90(1.08)$ & $-.19^{*}$ \\
\hline
\end{tabular}

\footnotetext{
${ }^{a}$ Welch statistic used; PCS: Pain Catastrophizing Scale; Beliefs about Epidural Analgesia Questionnaire: $1=$ completely disagree, $5=$ completely agree.$$
{ }^{*} p<.05 ;^{* *} p<.01 ;{ }^{* * *} p<.001 \text {. }
$$ 


\section{Results}

\subsection{Use of epidural analgesia, intention and demographic characteristics}

During pregnancy, $30.7 \%$ of the women were planning to use EA, $38.6 \%$ did not intend to use EA and about $30 \%$ had not made any decision yet. Eventually, more than half of the women $(55.3 \%, n=63)$ chose epidural analgesia. As might be expected, there was a strong relationship between intention and actual use of EA $\left(\chi^{2}(2)=24.15, p<.001\right)$. In about three-quarters of the women $(75.9 \%)$ the intention was realized. Only $27.3 \%(n=12)$ of the women who intended not to use EA, actually used EA. Only $20 \%(n=7)$ of the women who intended to use EA, did not use EA. About $65 \%$ $(n=23)$ of the women who had not decided, actually chose EA.

There was no difference in EA use between the two maternity clinics $\left(\chi^{2}(1)=0.57\right.$, ns). There were also no differences between the women who chose EA and the women who did not choose EA as a function of age $(F(1,112)=2.51, \mathrm{~ns})$, marital status $\left(\chi^{2}(3)=1.76, \mathrm{~ns}\right)$, profession $\left(\chi^{2}(5)=4.12\right.$, ns) and education level $\left(\chi^{2}(2)=1.31, \mathrm{~ns}\right)$. However, there were significant differences in EA use regarding parity (the number of children already given birth to). Women who were pregnant with their first child, more often chose EA than women who had already one or more children $\left(\chi^{2}(3)=7.95\right.$, $p<.05)$.

\subsection{Differences in beliefs about epidural analgesia}

The differences in beliefs about EA between women who actually chose EA and women who did not choose EA during childbirth were examined using one-way ANOVAs. Whenever the assumption of equal variances between groups was violated, the Welch statistic instead of the $F$-statistic was used. The differences between the two groups are presented in Table 1 . There were pronounced differences. In comparison with women who did not choose EA, those who chose EA expressed a greater desire to "enjoy their childbirth", "have a pain-free and relaxed childbirth", and they believed more strongly that "EA was comfortable and safe". These women also strongly agreed that "suffering during childbirth is needless", and were also more "afraid of being overwhelmed by the pain during childbirth". There were also significant effects of the immediate social context. In comparison with women who did not choose EA, women who chose EA, reported being more influenced by stories about contractions of other women, by positive experiences of family and friends, and by information from the maternity clinic. Overall, those who did not choose EA reported having more confidence in their ability to tolerate labour pain and reported being more able to give birth without EA than those who chose EA.

\subsection{Predictors of EA using logistic regression}

The unique value of the beliefs upon the actual use of EA was investigated using logistic regression analyses. Age, parity (number of children), catastrophizing about pain and the BEAQ items (except intention) were included as independent variables. A backward stepwise selection procedure was used to construct a logistic regression model and to calculate adjusted odds ratios (ORs) and confidence intervals (95\% CIs). Backward elimination begins with a complex model and sequentially removes terms. This procedure was preferred because it is often safer to delete terms from an overly complex model than to add terms to an overly simple one. Forward selection can stop prematurely because a particular test in the sequence has low power (Agresti, 2002). Correlation coefficients among the variables were computed to control for multicollinearity. Although there was a substantial correlation between age and parity $(r=.48)$, both variables were retained. In the final multivariate model variables with a $p$-value less than .05 were retained.

Table 2 summarizes the results of the final model. There were four unique predictors of EA. Parity reduced the odds of choosing EA by half. The desire to have a pain-free childbirth doubled the odds for EA. Fear of EA side effects reduced the odds of EA by half. Positive experiences with EA of family and friends had the most pronounced effect. All other variables were not significant, including catastrophizing about pain. This model proved to be parsimonious and robust. The fit of the model was also better than the fit of a model containing no predictors (null model: $\chi^{2}(4)=34.47, p<.0001$ ). The fit was not worse than a model containing all variables $\left(\chi^{2}(18)=6.15, \mathrm{~ns}\right)$. Our model also had good predictive qualities. Using the model, $71 \%$ of the women could be correctly classified as a function of their EA use. In contrast, the null model could only correctly classify $55 \%$ of the women. The robustness of our model was further investigated using two strategies. First, also a forward stepwise strategy was conducted. This strategy yielded comparable results. The only difference was related to the inclusion of an extra item: "the wish to fully enjoy childbirth". This item however does not add any new theoretical construct to the equation. Second, the model was validated using several random subsamples. All analyses yielded comparable models. We further evaluated the fit of the model using two additional statistics. First, the Hosmer-Lemeshow statistic was not significant $(p=.39)$, indicating that the model had an acceptable fit. Second, the Nagelkerke $R^{2}$ statistic showed that the likelihood rises to $34.9 \%$ when introducing the explanatory variables in our model. The Nagelkerke 
Table 2

Variables included in the derived model predicting the preference or non-preference for epidural analgesia

\begin{tabular}{lr}
\hline & OR \\
\hline Individual characteristics & $95 \%$ CI \\
Parity & $0.50^{*}$ \\
Attitudes & $2.24^{*}$ \\
I want a pain-free childbirth & $0.41^{* *}$ \\
I'm afraid of the potential side effects of EA & $1.18-4.28$ \\
Subjective norms & $2.77^{*}$ \\
Because of positive experiences with EA of family and friends, I'm thinking about choosing EA & $0.22-0.78$ \\
\hline
\end{tabular}

Note: $\mathrm{OR}=$ adjusted odds ratio; $95 \% \mathrm{CI}=95 \%$ confidence interval.

${ }^{*} p<.05 ;{ }^{* *} p<.01$.

$R^{2}$ statistic is an approximation of the explained variance $\left(R^{2}\right)$ concept for the ordinary regression model.

\subsection{The effect of pain catastrophizing upon use of EA and upon beliefs about EA}

To our surprise, pain catastrophizing was not predictive of EA use $(\operatorname{Wald}(1)=1.09, p=.30)$. For those women who chose EA, the mean PCS score was 17.67 $(\mathrm{SD}=8.45$; range $=3-33)$. For those women who did not choose EA, the mean PCS score was 16.00 (SD = 8.61; range 1-33). Further analysis showed that the group variances of the PCS scores were homogeneous (Levene $(1,112)=0.003, p>.05)$, indicating that the distributions of the PCS are comparable.

However, catastrophizing about pain was related to a number of beliefs about EA. The Pearson correlations between pain catastrophizing and beliefs are reported in Table 1. As expected, pain catastrophizing was positively related to the wish to fully enjoy childbirth, the view that suffering pain during childbirth is needless, the thought of not being able to tolerate pain during childbirth, and the fear of being overwhelmed by pain. Noteworthy was the finding that pain catastrophizing was positively related to the fear of pain during the insertion of the EA needle. Also of interest, was that pain catastrophizing was positively related to recommendations to use EA by the midwife and by the gynecologist.

\subsection{The effect of pain catastrophizing upon the childbirth experience}

Because it was expected that the use of EA would profoundly affect the experience during childbirth, the effect of pain catastrophizing was investigated separately for each group. Pearson intercorrelations between pain catastrophizing and the CEQ items were computed for both groups and are reported in Table 3. It was further tested whether the correlations differ between groups. The $Z$-values and $p$-levels that are associated with these differences, are also displayed in Table 3.

After childbirth, catastrophizing about pain was related to an increased intention to use EA on future occasions in both groups. Pain catastrophizing was also positively interrelated with a fear of pain during the beginning of the contractions in both groups. Although the pattern of correlations are different for women who

Table 3

Means $(M)$ and standard deviations (SD) for differences in the perceived experience of the childbirth for women who went through childbirth with or without epidural analgesia (EA). Pearson correlations between the Childbirth Experience Questionnaire items and pain catastrophizing for both groups of women and the difference between the correlations for both groups $(Z)$

\begin{tabular}{lccccc}
\hline Items of the Childbirth Experience Questionnaire & PCS EA & PCS non-EA & $Z$ & $M($ SD) with EA & $M($ SD) without EA \\
\hline I will choose EA in the future ${ }^{\mathrm{a}}$ & .25 & $.29^{*}$ & -0.22 & $4.42(1.01)$ & $2.41(1.43)^{* * *}$ \\
I was able to counter the contraction pain well & -.11 & $-.38^{* *}$ & 1.50 & $3.25(1.16)$ & $3.67(1.16)$ \\
The contraction pain was the worst pain I ever experienced & .08 & .11 & -0.15 & $3.58(1.28)$ & $3.85(1.39)$ \\
I imagined the pain to be less severe & .005 & .25 & -1.29 & $3.17(1.20)$ & $2.86(1.43)$ \\
I knew it would hurt, but not like this & .19 & $.53^{* * *}$ & $-2.05^{*}$ & $3.38(1.33)$ & $3.35(1.40)$ \\
When the contractions started I became afraid of the pain & $.37^{* *}$ & $.28^{*}$ & 0.52 & $2.67(1.32)$ & $2.12(1.42)^{*}$ \\
When I give birth again, I will have less fear of the pain & .005 & -.04 & 0.23 & $3.17(1.31)$ & $3.18(1.35)$ \\
Countering the painful contractions was easier than I expected & -.08 & -.19 & 0.58 & $2.94(1.34)$ & $3.22(1.35)$ \\
I expected the pain to be less frustrating & -.09 & .26 & -1.84 & $3.15(1.20)$ & $2.86(1.33)$ \\
Countering the contractions was harder than I had imagined & .07 & .27 & -1.07 & $3.81(1.12)$ & $3.31(1.33)^{*}$ \\
\hline
\end{tabular}

${ }^{\text {a }}$ Welch statistic used; PCS: Pain Catastrophizing Scale; Childbirth Experience Questionnaire: $1=$ completely disagree, $5=$ completely agree.

${ }^{*} p<.05 ;{ }^{* *} p<.01 ;{ }^{* * *} p<.001$. 
did choose EA and women who did not, only one significant difference emerged. For those who did not use EA, pain catastrophizing was related to a more overwhelming pain experience than for those who did choose EA.

\section{Discussion}

The present study was designed to investigate the reasons that might lead women to choose or not choose EA as a strategy for the management of pain during childbirth.

In this study $55 \%$ of the women used EA during childbirth. This percentage is high in comparison with data in the UK (20\%, Chamberlain et al., 1993) and Italy (33\%, Capogna et al., 1996), but comparable with other available statistics in Belgium (64\%, Cammu et al., 2003) and other Western countries such as the USA (50\%, Hawkins et al., 1997) and Israel (63\%, Horowitz et al., 2004). The large majority of women had decided in advance whether they would use EA. Twenty-five percent of women changed their mind. Similar percentages have been noted in previous studies (Ranta et al., 1995; McCrea, 1996; Goldberg et al., 1999), and indicate that efficient pain management during childbirth requires a flexible planning that allows for change (Wright et al., 2000). Changes in the intention to use EA are probably influenced by the first episodes of labour pain, its further course, and the experience or belief that one is (not) able to cope with pain (Wright et al., 2000).

The application of the theory of planned behaviour in explaining why women use EA during childbirth revealed a variety of specific attitudes, social influences and feelings of control that played a role in explaining the use of EA. Using a logistic technique, we were able to identify and develop a parsimonious and robust statistical model that allowed the prediction of EA use in $71 \%$ of the women. In that model, there were four unique predictors of EA: Parity status and the fear of the side effects of EA each reduced the odds of choosing EA by half, whereas the desire to have a pain-free childbirth and positive experiences with EA of family and friends each doubled the odds of choosing EA. It is apparent that the desire to have a pain-free childbirth is of paramount importance. To our surprise, health care providers such as midwives and gynecologists appeared to have no significant influence upon the decision. Probably, unless there are medical indications, health care providers leave the choice to women. More important were the social influences of the immediate environment such as other family members or friends who had positive experiences with EA. Fearing potential side effects of EA raised the threshold for EA use. As yet, it is not known why women underestimate or overestimate the risks of EA. An answer to this question may, however, be crucial in providing tailor-made and accurate information about the pros and cons of EA. Giving birth for the first time (primigravidae) doubled the odds for EA use. There may be several reasons for this finding. Having heard stories about the painful nature of childbirth from others, and the uncertainty about their own childbirth, may have made them overanxious and feeling less efficacious in coping with the pain. In line with this view, are the findings that multigravidae have more realistic expectations of labour pain, and feel efficacious in coping with labour pain (Lowe, 1992; McCrea et al., 2000).

Another aim of our study was to explore the effects of pain catastrophizing on EA and childbirth. We predicted that those who catastrophize about pain would be eager to avoid pain and thus would decide to use EA. This was not confirmed. However, the overall pattern of results regarding the interrelationship between pain catastrophizing on the one hand and specific beliefs about EA on the other hand was as expected. First, catastrophizing about pain was related to the fear of being overwhelmed by childbirth pain and to tendencies to avoid the pain (suffering pain is needless). In that respect, our findings add to accumulating evidence that catastrophizing about pain is strongly associated with fear of pain in pain-free volunteers (Crombez et al., 1998) and in chronic pain patients (Vlaeyen and Linton, 2000; Goubert et al., 2004). Second, catastrophizing about pain, especially in those who did not choose EA, was predictive of a distressing experience during contractions and childbirth. Our findings replicate and extend those of Wuitchik et al. (1990). In their study women with fear of pain, feelings of helplessness and loss of control experienced more pain during childbirth, and more distress-related thoughts during labour.

Why is pain catastrophizing not predictive of EA use? Our data indicate that the process of pain catastrophizing is more complicated than first thought. Pain catastrophizing seems to be related to an ambivalent position towards EA. On the one hand, those high in catastrophizing about pain feared becoming overwhelmed by the pain without EA. On the other hand, they were afraid of pain during the insertion of the EA needle. This ambivalent position towards EA might have obscured the relation between pain catastrophizing and use of EA. The ambivalence encountered by those high in catastrophizing about pain suggests that the relation between psychological variables and pain-related outcomes is not always straightforward. The deliberate and carefully weighing of the potential positive and negative consequences of each option may then result in a heightened uncertainty and indecisiveness, and may give rise to excessive contemplation and rumination. Facilitating the exploration of the pros and cons of each option may help this decisional imbalance (Miller and Rollnick, 2002). 
Of further interest to this study are the findings regarding the relationship between pain catastrophizing and advice from others. The correlational analysis revealed that pain catastrophizing was related to a pronounced perceived impact of others upon the decision to use EA. Those who catastrophize about pain were sensitive to the recommendations of the midwife and the gynecologist. There are many possible explanations for this finding. An intriguing explanatory model is in terms of the communicative function of catastrophizing. Sullivan et al. (2001) extended the appraisal view of pain catastrophizing and urged researchers to look at its communicative function as a signal of distress to solicit assistance from others. Although our findings reveal that pain catastrophizing has a social impact, it remains unclear how this social process takes place. It may be possible that others provide advice because they feel that women who catastrophize about pain are undecided, and experience distress and fear about childbirth pain. In that respect the social function of pain catastrophizing is unintentional and related to observable signs of distress. It may also be possible that women who catastrophize, share their feelings of distress with others, and specifically request help and advice from the immediate environment or from health care providers. In this sense the social function of pain catastrophizing is an intentional and goal-directed form of coping (Severeijns et al., 2004). Both experimental and clinical studies are needed to further explore these social processes in pain catastrophizing (see Goubert et al., 2005).

There are some limitations to this study. First, although the response rate was adequate $(51 \%)$, it is not known why a large minority declined to participate. Second, we used an estimation strategy to replace a number of missing data. Although our algorithm provides relatively unbiased and robust estimates of missing values (Schafer and Graham, 2002), these can never replace real-life values. Third, we have no information regarding the use of EA during previous childbirth(s). This might have been useful in understanding why parity is an important predictor of actual EA use. Fourth, the experience of childbirth pain was not assessed using standard instruments of pain (Melzack, 1984). Fifth, the theory of planned behaviour is an expectancy-value model (Conner and Sparks, 1996). In line with previous studies, we mainly focused upon the expectancy/belief component. There may be some merit in addressing separately the expectancy and value component. Sixth, one should be mindful of the difference between statistical and clinical significance. Most differences reported here have a moderate effect size, and may not be directly transferred into a clinical context. Finally, although a prospective design was used, our findings may at best be regarded as predictive indicators. Further studies should focus upon the underlying mechanisms and in particular upon the social impact of pain catastrophiz- ing. In that area there remains a need for specific models that allow differential hypotheses and model testing.

\section{Acknowledgements}

The authors wish to thank Tania Van den Hove for the collection of the data. The authors also want to thank the staff of the two participating hospitals for their help with the recruitment of the patients.

\section{References}

Agresti A. Categorical data analysis. second edition. New York: Wiley; 2002.

Ajzen I. The theory of planned behavior. Organ Behav Hum Decis Process 1991;50:179-211.

Aldrich S, Eccleston C, Crombez G. Worrying about chronic pain: vigilance to threat and misdirected problem solving. Behav Res Ther 2000;38:457-70.

Armitage CJ, Conner M. Efficacy of the theory of planned behavior: a meta-analytic review. Br J Soc Psychol 2001;40:471-99.

Cammu H, Martens G, De Coen K, Van Mol C, Defoort P. Perinatale activiteiten in Vlaanderen 2003. Brussel: SPE; 2003.

Capogna G, Alahuhta S, Celleno D, De Vlieger H, Moreira J, Morgan $\mathrm{B}$, et al. Maternal expectations and experiences of labour pain and analgesia: a multicentre study of nulliparous women. Int $\mathbf{J}$ Obstet Anesth 1996;5:229-35.

Caton D. Medical science and social values. Int J Obstet Anesth 2004;13:167-73.

Caton D, Frölich MA, Euliano TY. Anesthesia for childbirth: controversy and change. Am J Obstet Gynecol 2002;186:S25-30.

Chamberlain G, Wright A, Steer P. Pain and its relief in labour: report of the 1990 NBT survey. Edinburgh: Churchill Livingstone; 1993.

Conner M, Sparks P. The theory of planned behaviour and health behaviours. In: Conner M, Norman P, editors. Predicting health behaviour: research and practice with social cognition models. Buckingham: Open University Press; 1996. p. 121-62.

Crombez G, Eccleston C, Baeyens F, Eelen P. When somatic information threatens, pain catastrophizing enhances attentional interference. Pain 1998;75:187-98.

Crombez G, Van Damme S, Eccleston C. Hypervigilance to pain: an experimental and clinical analysis. Pain 2005;116:4-7.

Dempster AP, Laird NM, Rubin DB. Maximum likelihood estimation from incomplete data via the EM algorithm. J Royal Stat Soc 1977;B39:1-38.

Devries H, Backbier E, Kok G, Dijkstra M. The impact of social influences in the context of attitude, self-efficacy, intention, and previous behavior as predictors of smoking onset. J Appl Soc Psychol 1995;25:237-57.

Godin G, Kok G. The theory of planned behavior: a review of its applications to health-related behaviors. Am J Health Promot 1996;11:87-98.

Goldberg AB, Cohen A, Lieberman E. Nulliparas' preferences for epidural analgesia: their effects on actual use in labor. Birth 1999;26:139-43.

Goubert L, Crombez G, Van Damme S. The role of neuroticism, pain catastrophizing and pain-related fear in vigilance to pain: a structural equations approach. Pain 2004;107:234-41.

Goubert L, Craig KD, Vervoort T, Morley S, Sullivan MJL, Williams $\mathrm{ACdeC}$, et al. Facing others in pain: the effects of empathy. Pain 2005;118:285-8. 
Hawkins JL, Gibbs CP, Orleans M, Martin-Salvaj G, Beaty B. Obstetric Anesthesia Work Force Survey, 1981 versus 1992. Anesthesiology 1997;87:135-43.

Horowitz ER, Yogev Y, Ben-Haroush A, Kaplan B. Women's attitudes toward analgesia during labor - a comparison between 1995 and 2001. Eur J Obstet Gynecol Reprod Biol 2004;117: $30-2$.

Keefe FJ, Rumble ME, Scipio CD, Giordano LA, Perri LM. Psychological aspects of persistent pain: current state of the science. J Pain 2004;5:195-211.

Leighton BL, Halpern SH. The effects of epidural analgesia on labor, maternal, and neonatal outcomes: A systematic review. Am J Obstet Gynecol 2002;186:S69-77.

Little RJA, Rubin DB. The analysis of social science data with missing values. Sociological Methods and Research 1989;18:292-326.

Lowe NK. Differences in first and second stage labor pain between nulliparous and multiparous women. J Psychosom Obstet Gynaecol 1992;13:243-53.

McCrea BH. An investigation of rule-governed behaviours in the control of pain management during the first stage of labour. Unpublished DPhil Thesis, University of Ulster, 1996.

McCrea H, Wright ME, Stringer M. Psychosocial factors influencing personal control in pain relief. Int J Nurs Stud 2000;37: 493-503.

Melzack R. The myth of painless childbirth. Pain 1984;19:321-37.

Miller WR, Rollnick S. Motivational interviewing: preparing people for change. New York: Guilford Press; 2002.

Ranta P, Spalding M, Kangas-Saarela T, Jokela R, Hollmén A, Jouppila $\mathrm{P}$, et al. Maternal expectations and experiences of labour pain - options of 1091 Finnish parturients. Acta Anaesthesiol Scand 1995;39:71-8.

Reynolds F, Sharma SK, Seed PT. Analgesia in labour and fetal acidbase balance: a meta-analysis comparing epidural with systemic opioid analgesia. Br J Obstet Gynaecol 2002;109:1344-53.

Schafer JL, Graham JW. Missing data: our view of the state of the art. Psychol Methods 2002;7:147-77.

Severeijns R, Vlaeyen JWS, van den Hout MA. Do we need a communal model of pain catastrophizing? An alternative explanation. Pain 2004;111:226-9.

Sullivan MJL, Bishop SR, Pivik J. The pain catastrophizing scale: development and validation. Psychol Assess 1995;7:524-32.

Sullivan MJL, Thorn B, Haythornthwaite JA, Keefe F, Martin M, Bradley LA, et al. Theoretical perspectives on the relation between catastrophizing and pain. Clin J Pain 2001;17:52-64.

Sullivan MJL, Tripp DA, Santor D. Gender differences in pain and pain behavior: the role of catastrophizing. Cog Ther Res 2000;24:121-34.

Van Damme S, Crombez G, Bijttebier P, Goubert L, Van Houdenhove B. A confirmatory factor analysis of the pain catastrophizing scale: invariant factor structure across clinical and non-clinical populations. Pain 2002;96:319-24.

Vlaeyen JWS, Linton SJ. Fear-avoidance and its consequences in musculoskeletal pain: a state of the art. Pain 2000;85:317-32.

Wright ME, McCrea H, Stringer M, Murphy-Black T. Rule-governed behaviours: reflections from practice. Clinical effectiveness in nursing 2000;4:128-37.

Wuitchik M, Hesson K, Bakal DA. Perinatal predictors of pain and distress during labor. Birth 1990;17:186-91. 